\title{
RULE OF LAW Y CASOS RECALCITRANTES*
}

\section{RULE OF LAW AND RECALCITRANT CASES}

\author{
Pau LUQUE**
}

\section{Resumen:}

Según una sofisticada versión del llamado rule of law, las reglas del derecho deben ser generales, en el sentido de que deben regular clases de casos, no situaciones particulares. La posibilidad de prever cuáles van a ser las decisiones jurídicas depende, entre otras cosas, de este carácter general de las normas. Pero junto a la previsibilidad y certeza que aporta, el carácter general de las reglas puede tener un reverso negativo: en algunas ocasiones, cuando se aplican a algunas circunstancias particulares, lo que parecía funcionar de forma satisfactoria en la teoría o en abstracto, proporciona soluciones inadmisibles (inadmisibles con arreglo a alguna concepción de la justicia). Esos casos son conocidos como casos recalcitrantes. En este artículo propongo una manera de entender el razonamiento jurídico-práctico que mitiga ese reverso negativo de las reglas generales conservando buena parte de la certeza que aportan.

\section{Palabras clave:}

Estado de derecho; decisión jurídica; casos recalcitrantes; razonamiento jurídico.

\section{Abstract:}

According to a sophisticated version of the Rule of Law, legal rules should be general in aiming at regulating classes of cases instead of concrete situations. The possibility to foresee which legal decisions will be reached depends on

* Artículo recibido el 9 de agosto de 2019 y aceptado para su publicación el 11 de noviembre de 2019.

** Instituto de Investigaciones Jurídicas de la UNAM, pls2001@gmail.com.

Problema. Anuario de Filosofía y Teoría del Derecho, núm. 14, enero-diciembre de 2020, pp. 217-246 Ciudad de México, ISSN 2007-4387, se distribuye bajo una Licencia Creative Commons Reconocimiento-No Comercial-Sin Derivados 4.0 Internacional (CC BY-NC-ND 4.0). 
PAU LUQUE

the general character of norms. However, along with the predictability and certainty it provides, the generality of rules can have a negative reverse side: when applied to certain particular circumstances - what seemed to function satisfactorily in abstract - provides inadmissible solutions according to some conception of justice. Those cases are known as recalcitrant cases. In this article, I suggest a way of understanding the practical legal reasoning which mitigates the general rules' negative reverse side while preserving an important part of the certainty they provide.

Keywords:

Rule of Law; Legal Decision; Recalcitrant Cases; Legal Reasoning. 
SUMARIO: I. Introducción. II. Rule of law y casos recalcitrantes. III. Modelos de razonamiento jurídico-práctico para la toma de decisiones. IV. Objeciones. V. Conclusión. VI. Bibliografía.

\section{INTRODUCCIÓN}

¿Es deseable que el derecho guíe el comportamiento humano, tanto el de los ciudadanos como el de los poderes públicos, mediante un sistema de reglas? Según el ideal del rule of law (en adelante, RoL), la respuesta a esa pregunta es positiva (Fuller, 1969: 106). El RoL, además, está comprometido con que las reglas del derecho deben ser generales, esto es, deben regular clases de casos, no situaciones particulares. Hay varias razones que justifican esta preferencia por reglas generales, pero la más relevante, a los efectos de este artículo, descansa en el hecho de la previsibilidad de las soluciones jurídicas; si sé por avanzado qué clase de acciones están prohibidas y cuáles están permitidas puedo trazar un plan de vida. La cualidad de generalidad - junto con otras, como la publicidad de las reglas y el carácter no retroactivo de las mismas- es lo que me permite saber con anticipación qué está prohibido y qué está permitido y, en consecuencia, las reglas generales me permiten, para usar una aguda expresión de Raz, ser el autor de mi propia vida.

Sin embargo, junto con ese deseo, muchos juristas hacen notar que cuando las reglas generales entran en contacto con las circunstancias de los casos particulares, en algunas ocasiones la solución normativa que arroja la regla general proporciona soluciones inaceptables. Esto ocurriría debido a que la naturaleza general de las mismas (i. e. el hecho de que regulan clases de casos) hace que las reglas sean sobre e infrainclusivas, ${ }^{1}$ incluyen más casos de los que deberían incluir y no incluyen casos que sí deberían incluir.

¿Es posible armonizar ambas preocupaciones? ¿Es posible decidir sobre la base de reglas generales y al mismo tiempo decidir sobre la

1 Schauer, F., Playing by the Rules. A Philosophical Examination of Rule-Based Decision-Making in Law and in Life, OUP, 1991, pp 31-34. 
base de las circunstancias del caso particular? Dicho de otro modo: ¿es posible conservar las virtudes que instancia el ideal del RoL atendiendo y ponderando las circunstancias de cada caso particular?

A primera vista, el ideal del RoL es incompatible, o por lo menos está en severa tensión, con la consideración caso por caso que exigiría hacer frente al carácter sobre e infrainclusivo de las reglas generales. Decidir atendiendo a las circunstancias de los casos particulares significa disminuir la previsibilidad de las decisiones judiciales. ${ }^{2}$ Así que parece que o bien tenemos reglas generales pagando el precio de tener algunas soluciones inaceptables o bien decidimos en cada caso particular pagando el precio de perder previsibilidad de las soluciones. ¿Hay alguna manera de armonizar estos dos deseos? Creo que hay una manera de hacerlo que minimiza el precio que se paga en cada una de las posibilidades. Esto es lo que intentaré mostrar en este artículo.

Procederé del siguiente modo: en la primera sección, presentaré el ideal del RoL tal y como será entendido aquí. En esta misma sección desarrollaré, aunque de forma breve, el fenómeno y concepto de los casos recalcitrantes. En la segunda sección, sobre la base de tres niveles distintos del razonamiento jurídico-práctico, delinearé tres posibles modelos del razonamiento jurídico-práctico. Será en esta sección donde plantearé mi propuesta orientada a armonizar las preocupaciones anteriormente mencionadas. En la tercera sección, tomaré en consideración algunas objeciones que podrían dirigirse contra mi propuesta, e intentaré dar respuesta a ellas. Por último, concluiré.

\section{RULE OF LAW Y CASOS RECALCITRANTES}

\section{Rule of Law}

El RoL es ante todo una forma de gobierno que promete protección e instrumentos de tutela contra las potenciales o reales arbitrarie-

2 Kramer, M., Objectivity and the Rule of Law, CUP, 2007, p. 110; Maccormick, N. Rhetoric and the Rule of Law, OUP, 2005, p. 79; Caracciolo, R., El derecho desde la filosofía, Madrid, CEPC, 2009, p. 259; Raz, J., The Authority of Law, OUP, 1979, p. 219; Waldron, J., "Stare Decisis and the Rule of Law: A Layered Approach", 111 Michigan Law Review 1, 2012, pp. 18 y 32. 
dades del ejercicio de poderes. ${ }^{3}$ Para evitar la arbitrariedad del poder, el ideal del RoL exige una serie de requisitos formales que las normas jurídicas - tanto si están dirigidas a los poderes públicos como si están dirigidas a los ciudadanos- deben poseer: ${ }^{4} 1$ ) generalidad; 2 ) publicidad; ${ }^{5}$ 3) inteligibilidad y claridad; 4) relativa estabilidad; 5) prospectividad y no retroactividad; 6) coherencia entre ellas, y 7) que no prescriban algo imposible de cumplir.

El respeto a estos requisitos formales se considera normalmente como algo moralmente deseable, pero no es considerado el único criterio de evaluación moral de un ordenamiento jurídico. ${ }^{6}$ Pero en todo caso estas exigencias aseguran un mínimo grado de autonomía personal y de dignidad a los ciudadanos. ${ }^{7}$ ¿En qué sentido? Los ciudadanos, cuando las reglas cumplen esos requisitos formales, pueden entender el contenido de la regla jurídica y prever las consecuencias de cumplir y también de violar la regla; los ciudadanos pueden tomar una decisión habida cuenta de todo - all things considered - de forma autónoma; los ciudadanos pueden, en fin, hacer planes de vida (dentro del paisaje jurídico en el que están). Dicho de manera más sintética: los destinatarios de las normas pueden formarse expectativas razonables acerca de las consecuencias de sus propias acciones, y también pueden for-

3 Postema, G., "Il dominio del diritto", en Pino, G. y Villa V. (eds.), Rule of Law. L'ideale della legalità, Bologna, Il Mulino, 2016, p. 92. Caracciolo, op. cit., 2009, p. 252.

No presentaré el RoL en términos de la contraposición entre "gobierno de los hombres" y "gobierno de las leyes" porque como han señalado algunos autores (Celano, 2015; Barberis, 2016: 13) esta contraposición se debilita mucho cuando uno toma en cuenta que son los hombres quienes hacen, interpretan y aplican las leyes.

4 Celano, B., "Rule of Law y particularismo ético", en Luque, P. (ed.), Particularismo. Ensayos de filosofía del derecho y filosofía moral, Madrid, Marcial Pons, 2015, p. 152; Kramer, op. cit., 2007, cap 2. Raz, op. cit. 1979, pp. 215-218.

5 Qué significa exactamente esta exigencia, sin embargo, no está claro. Véase Celano (2013).

6 Pino, G., "Legalità penale e Rule of law", en Pino, G. y Villa, V. (eds.), Rule of law. L'ideale della legalità, Bologna, Il Mulino, 2016, p. 214.

7 Waldron, J., "How Law Protects Dignity", Cambridge Law Journal, 71, 2a. ed., 2012, pp. 200-222. 
marse expectativas razonables acerca de cómo se comportarán los otros ciudadanos. ${ }^{8}$

Así pues, el RoL busca, entre otras cosas, ofrecer certeza o previsibilidad. Todas las exigencias mencionadas anteriormente contribuyen a que los ciudadanos sepan por avanzado cuál será la respuesta probable de las instituciones jurídicas a sus acciones. ${ }^{9}$

En este artículo querría centrarme en el primer elemento de esa lista de requisitos formales, esto es, el carácter general de las reglas jurídicas. Una de las razones por la que los partidarios del RoL en este sentido prescriben que las reglas jurídicas deben ser generales es justamente porque proporcionan certeza. ${ }^{10}$

¿Cómo funcionaría el argumento que muestra esto último?

Imaginémonos que una regla jurídica afirma que cuando ocurran las circunstancias $p, q$ y $r$, entonces será obligatorio el acto $x$. Esto es una manera distinta de decir que en aquellas situaciones en que concurran las circunstancias $p, q$ y $r$, el derecho regula esos casos con la consecuencia $O x$. Yo, que soy destinatario de esa regla, puedo saber ex ante que si se dan las circunstancias $p, q$ y $r$, entonces la consecuencia jurídica prevista por el derecho es $O x$. Si consigo identificar instancias particulares de las condiciones generales $p, q$ y $r$, entonces puedo saber que la decisión deberá ser $0 x$. Es el carácter general -así como la capacidad del destinatario de identificar instancias particulares de las condiciones generales de aplicación- lo que permite saber cuál será la consecuencia jurídica por anticipado. Si no existieran —o no fueran públicas - esas condiciones generales de aplicación, no podría saber cuándo la decisión sería $O x$ o cuándo sería no-Ox.

8 Pino, G., "Legalità penale e Rule of law", en Pino, G. y Villa, V. (eds.), Rule of law. L'ideale della legalità, Bologna, Il Mulino, 2016, p. 216.

9 Tamanaha, B., "A Concise Guide to the Rule of law", en Palombella, G. y Walker, N., (eds.), Relocating the Rule of Law, Oxford, Hart, 2009, p. 7.

10 Ésta no es la única virtud del carácter general de las reglas. Por ejemplo, las reglas respetan el principio de igualdad, esto es, tratan igual casos iguales (Schauer, 1991: 135). Sin embargo, lo que pone justamente en tela de juicio la existencia de casos recalcitrantes es que en realidad las reglas generales esconden el hecho de que algunos casos que parecen iguales en realidad no lo son. Volveré más tarde sobre ello. 


\section{Casos recalcitrantes y Rule of Law}

Así pues, el RoL nos dice que nada asegura mejor la previsibilidad de las decisiones de los jueces y otros órganos de aplicación del derecho que las reglas generales (y públicas y prospectivas). En este sentido, las reglas conforman un entorno social e institucional que nos permite calcular qué ocurrirá si hacemos tal o cual acción. ${ }^{11}$

Pero ¿qué ocurre cuando esas reglas generales entran en contacto con el entorno fáctico de los casos particulares? Muchas veces, se dirá, no ocurre nada llamativo: simplemente aplicamos la regla general al caso particular, obtenemos la solución, y todo parece estar en orden. Dicho de un modo distinto, parece que las reglas generales normalmente funcionan bien: obtenemos de ellas lo que queremos. ${ }^{12}$

Pero imaginémonos ahora una regla que diga "prohibida la entrada de perros al restaurante". Y supongamos ahora que una persona ciega, acompañada de un perro lazarillo, pretende entrar al restaurante. De la aplicación estricta de la regla se sigue que el perro lazarillo, y por lo tanto la persona ciega, no pueden entrar en el restaurante. Pero esta situación nos parece inaceptable. Este tipo de casos son conocidos como casos recalcitrantes. Es en estos casos donde se pone de manifiesto la denominada infra y sobreinclusión de la regla general. ${ }^{13}$

La única manera de hacer frente a ese rasgo infra y sobreinclusivo de las reglas generales es atendiendo a las circunstancias particulares del caso. Pero si hacer frente a los resultados injustos que flore-

11 Waldron, J., "The Concept and the Rule of Law", Georgia Law Review, 43, 2008, pp. 23 y 55, núm. 173.

Von Hayek trazaba incluso una analogía entre las leyes de la naturaleza y las leyes jurídicas, en el sentido de que estas últimas fijaban las características en las cuales el individuo se puede mover, Véase Von Hayek (1969).

12 "Normalmente" denota, en este contexto, lo que ocurre la mayor parte de las veces, Celano (2012).

13 Schauer, F., Playing by the Rules. A Philosophical Examination of Rule-Based Decision-Making in Law and in Life, OUP, 1991, pp. 31-34.

Un ejemplo de la infrainclusión consistiría en que la regla no impediría la entrada de osos al restaurante (Rodríguez, 2012: 128). 
cen en los casos recalcitrantes exige abandonar el carácter general de las reglas, parece que el derecho renuncia a una de las virtudes principales de regirse mediante el RoL, esto es, la certeza o la previsibilidad de las consecuencias normativas de nuestras acciones.

Así, parece que nos enfrentamos a un dilema: o bien abandonamos la generalidad de las reglas, perdiendo certeza aunque ganando, por hipótesis, justicia, o bien nos mantenemos fieles al carácter general de las reglas, conservando certeza, pero perdiendo, por hipótesis, justicia. ${ }^{14}$ ¿Es posible salir indemne de este dilema? Creo que no. Sin embargo, creo que hay una manera de minimizar los costes de ambos cuernos del dilema. Pero antes de abordar esta posibilidad, o, mejor dicho, para abordar esa posibilidad, debo delinear el modelo de razonamiento jurídico-práctico que usaré para ofrecerla.

\section{MODELOS DE RAZONAMIENTO JURÍDICO-PRÁCTICO PARA LA TOMA DE DECISIONES}

El razonamiento jurídico-práctico está dividido en tres niveles, ${ }^{15}$ que podemos denominar: nivel más profundo o de las razones, nivel medio o de las formulaciones deónticas y nivel menos profundo o de las soluciones normativas del caso particular.

El nivel más profundo está constituido por las razones, o, mejor dicho, por el balance de razones, a favor o en contra de una cierta acción. Este balance de razones justifica, o se cristaliza, en el segundo nivel: el balance de razones se transforma en una formulación deóntica, es decir, en lo que normalmente denominamos una regla (o una norma). Esta formulación deóntica tiene la forma de un condicional

14 Conviene aclarar que cuando digo que el RoL demanda certeza no quiero decir que exige que todas las decisiones sean predecibles. Entiendo que la certeza es gradual y cuando en la formulación del dilema hablo de perder o conservar certeza, me refiero a perder o conservar grados de certeza. En este sentido, el dilema se plantea para el RoL en referencia al grado máximo de certeza posible (posible en relación no con los casos recalcitrantes - porque en eso consiste justamente el dilema-, sino en relación, por ejemplo, con casos de indeterminación lingüística de ambigüedad o vaguedad).

15 Sigo aquí, con ciertas libertades, a Schauer (1991), Raz (1975) y Celano (2015). 
el antecedente del cual contiene las condiciones fácticas generales cuyo acaecimiento implica una consecuencia normativa (Redondo, 2005). Esta consecuencia normativa se proyecta en el tercer nivel, en que esa consecuencia normativa resulta ser la solución para un caso particular.

Llamaré a este modelo el modelo estándar.

Este modelo estándar de razonamiento para la toma de decisiones puede ser todavía un poco más especificado. Llamaré a esta especificación modelo de la toma de decisiones basado en reglas. Tal especificación ha sido criticada, por lo que llamaré modelo particularista de la toma de decisiones.

A continuación presentaré estos dos modelos, y, tras ello, presentaré mi propia propuesta, que denominaré modelo intermedio, y que estará destinada a enmendar las debilidades tanto del modelo basado en reglas como del modelo particularista.

Para el modelo de la toma de decisiones basado en reglas, tomar decisiones significa aplicar la regla general al caso particular, ${ }^{16}$ es decir, este modelo afirma el carácter subsuntivo de la actividad de toma de decisiones. ${ }^{17}$ Para este modelo, el primer nivel del razonamiento es irrelevante para el momento de la toma de decisiones (aunque no, por supuesto, a la hora de justificar la regla). Es irrelevante, porque si a la hora de tomar decisiones atendemos a las razones cuya cristalización es la regla (en el sentido de la formulación deóntica), entonces la regla pasa a ser inútil, un ornamento de más, ya que lo que estaríamos haciendo al tomar una decisión en un caso particular es aplicar directamente las razones que justifican la regla, con lo cual esta última sería, en el mejor de los escenarios, redundante. Las reglas sólo sirven, según el modelo de la toma de decisiones basado en reglas, si son opacas respecto del nivel de las razones. ${ }^{18}$

16 Esta presentación podría inducir a pensar que el mencionado modelo cae en algún tipo de formalismo jurídico, lo cual, a pesar de que la conexión no es completamente infundada, es inexacto. Sobre los diversos equívocos y ambigüedades del formalismo jurídico, véase Schauer (1988).

17 Shafer-Landau, R., “Moral Rules”, Ethics 107, Julio de 1997, pp. 586 y 587.

18 Esta presentación del modelo, como es sabido, está ligada al modelo de la autoridad jurídica como servicio de $\operatorname{Raz}(1979,2006)$. 
Para el modelo particularista de toma de decisiones, en cambio, tomar decisiones no consiste en inferir la decisión a partir del caso general, ${ }^{19}$ la toma de decisiones consiste en valorar, en cada caso particular, si las razones que habitan en el primer nivel se aplican o no al caso particular (es decir, en el tercer nivel). Este modelo particularista afirma justamente aquello que el modelo basado en reglas quería evitar, a saber: que las reglas pueden resultar superfluas, que lo relevante para la toma de decisiones son las razones de primer nivel, y que éstas son las que deben ser reevaluadas para cada caso particular, porque sólo ello nos asegurará tomar la decisión más justa posible.

Obsérvese que el modelo basado en reglas es rígido respecto de todos los niveles del modelo estándar: en la toma de decisión no se puede modificar el balance de razones (porque está vetado que el decisor acuda a él) ni la formulación de la regla. Para el modelo particularista, en cambio, se puede (y se debe, si lo que se quiere es alcanzar una decisión justa) modificar el balance de razones de un caso a otro, y también la formulación deóntica, aunque ésta, en realidad, pasa a ser una expresión prácticamente vacía de contenido hasta que entra en contacto con el ambiente fáctico del caso particular.

Se trata, naturalmente, de versiones simplificadas de estos dos modelos. Pero hay propuestas muy elaboradas que están en la misma órbita de estos modelos. En general, los llamados positivistas normativos o éticos sostienen concepciones del derecho, o por lo menos de la aplicación de las normas jurídicas, en que las reglas generales son -o deberían ser, pues se trata de posiciones regulativas- el elemento clave (o incluso único). ${ }^{20}$

19 Celano, B., "Rule of law y particularismo ético", en Luque, P. (ed.), Particularismo. Ensayos de filosofía del derecho y filosofía moral, Madrid, Marcial Pons, 2015, p. 156.

Dancy, J., “QQué es el particularismo en ética”, en Luque, P. (ed.), Particularismo. Ensayos de filosofía del derecho y filosofía moral, Madrid, Marcial Pons, 2015, p. 31.

Dancy es particularmente radical en este punto, puesto que no sólo niega la posibilidad de la inferencia deductiva cuando se trata por lo menos del juicio moral, sino también de la inferencia no deductiva, Dancy (2015: 31 y 32).

20 Laporta, F., El imperio de la ley. Una visión actual, Madrid, Trotta, 2007, cap. V-VI, y Campbell, T., The Legal Theory of Ethical Positivism, Syndey, Darmouth, Aldershot/Brookfield/Singapore, 1996. 
Por su parte, el modelo particularista ha sido desarrollado ampliamente por el propio Bruno Celano. Pero en la misma familia de teorías particularistas, también podría inscribirse - con cierta flexibilidad conceptual, lo admito- la concepción de la adjudicación judicial que Cass Sunstein denominó "minimalismo judicial". Esta teoría, a pesar de que no se apoya en el aparato teórico del particularismo, invoca el dicho "one case at a time" para defender que las decisiones judiciales (al menos las de la Suprema Corte) resuelvan únicamente los casos particulares que le lleguen y desista de formular soluciones para casos - o reglas - generales. ${ }^{21}$ La razón es doble: por un lado, política, porque Sunstein considera que los contenidos de las reglas generales deben ser el producto de procesos democráticos; y, por otro lado, teórica, porque la Corte no puede anticipar el alcance y las consecuencias de sus propias decisiones en el futuro, ya que presupone $-\mathrm{y}$ aquí es donde el minimalismo judicial revela su naturaleza particularista- que no es posible conocer de antemano y con exhaustividad las circunstancias del caso particular.

Ambos modelos, el de las reglas y el particularista, pueden ser, y de hecho han sido, refinados. Pero a los efectos de la discusión en la que quiero intervenir con este artículo, creo que es suficiente con el esbozo que he trazado aquí.

Sin embargo, creo que hay una tercera posibilidad, que denominaré "modelo intermedio", y que se diferencia del modelo basado en reglas, porque: 1) admite que se puede modificar la formulación de las reglas, y del modelo particularista porque 2) afirma que el balance de razones no se modifica en virtud de las circunstancias particulares del caso.

Para el modelo intermedio, la mejor toma de decisiones se hace matizando el modelo de razonamiento estándar del siguiente modo: en el nivel más profundo se encuentra el balance general de razones a favor o en contra de una acción; este balance queda tentativamente cristalizado en una regla, cuyo antecedente fáctico puede ser

21 Sunstein, C., One Case at a Time. Judicial Minimalism on the Supreme Court, HUP, 2001. 
modificado para que se conforme al balance de razones del primer nivel; y el tercer nivel está compuesto por la solución al caso particular, fruto de la subsunción del caso particular en el supuesto de hecho general previsto por la regla (y, en caso necesario, de la modificación de la regla). ${ }^{22}$

Pongamos el famoso caso de la regla "prohibida la entrada de perros en el restaurante" y el supuesto de la persona ciega que quiere entrar con un perro lazarillo al restaurante. Analicémoslo desde el punto de vista de los tres modelos de toma de decisión e imaginémonos que hay tres guardias que toman decisiones basándose en cada uno de los modelos delineados hasta el momento.

Guardia que toma su decisión apoyándose en el modelo basado en reglas. El guardia simplemente verifica que un perro quiere entrar al restaurante y aplica la solución prevista por la regla. Si hay un perro

22 Soy consciente de la existencia de lo que podrían llamarse otros modelos intermedios, en el sentido de modelos que también se sitúan entre el modelo de las reglas y el modelo particularista. Una comparación detallada de las diferencias con el modelo intermedio que yo estoy presentando aquí deberá esperar otra ocasión. En este contexto, me limitaré a señalar algunas diferencias básicamente de enfoque. Por ejemplo, podemos encontrar aquellos modelos que se inscriben en la tradición de Normative Systems, de Alchourrón y Bulygin, y de la lógica deóntica derrotable (Alchourrón, 1996). Con este tipo de modelos, creo que la diferencia descansa en que su motivación está en encontrar acomodo técnico al fenómeno de los casos recalcitrantes, es decir, su preocupación parece ser mapear el segundo nivel del razonamiento práctico de forma racional, mientras que mi interés principal tiene más que ver con el nivel más profundo, el nivel del balance de razones. A mí me importa poner énfasis en el carácter general del balance de razones, y no tanto —al menos no tanto como esos otros modelos intermedios alternativos- de las formulaciones deónticas.

Otro modelo, el de Dworkin $(1977,1986)$, se sitúa entre el modelo de las reglas y el del particularismo. Sin embargo, el modelo de Dworkin es singular, porque parece que pretende alcanzar para el derecho lo mismo que el modelo particularista -es decir, justicia-, pero no con el andamiaje conceptual particularista, sino con los principios normativos (generales) imbricados en el derecho. Por lo demás, y como se verá, qué razones son relevantes depende, en mi modelo, de un elemento fáctico típicamente positivista, como lo es la convención de los officials, mientras que en el modelo de Dworkin, la convención jurídica o es irrelevante o tiende a tener un papel más bien marginal cuando de lo que se trata es de encontrar la right answer a cada caso particular. 
(no importa que sea un perro lazarillo), negará la entrada; si no, no. Y así hará en todos los casos. ${ }^{23}$

Guardia particularista. El guardia entra a valorar si, en este caso particular, la solución aportada por la regla es conforme al balance de razones que justifica la regla. El balance de razones que justifica la regla nos dice que es deseable no molestar a los clientes del restaurante con animales, pero los perros lazarillos están adiestrados precisamente para que puedan convivir en ambientes humanos de forma discreta, es decir, para que no molesten. Así que el guardia particularista llega a la conclusión de que la aplicación de la regla tal y como está formulada a este caso particular no es consistente con el balance de razones que la justifica, y, por lo tanto, resuelve de forma incompatible con como lo haría el guardia que basa su decisión en la regla, es decir, en este caso el guardia particularista deja entrar al perro al restaurante. Pero el guardia particularista hará esta valoración para todo caso particular en el futuro, tanto si se parece a éste como si no, pues el balance de razones sólo sirve para este caso particular. 0 , dicho de un modo algo distinto, el balance de razones cuantifica sobre casos particulares, no sobre clases de casos. Por ello, el guardia particularista considera que aunque la regla puede modificarse para incluir el nuevo caso, no vale la pena hacerlo, porque a fin de cuentas lo que aplicamos - lo único que podemos aplicar si queremos obtener una solución justa- en el caso particular es el balance de razones.

Guardia intermedio. El guardia intermedio procede, en este caso particular concreto, como el guardia particularista: valora si la solución aportada por la regla en este caso particular es consistente con el balance de razones que justifica la regla y, por hipótesis, llega a la misma conclusión que su par particularista. Sin embargo, hay una diferencia fundamental con aquél: el balance de razones - "es deseable no molestar a los clientes con animales" - no opera sobre un caso particular, sino sobre el caso general. Dicho de otro modo, el balance de razones no vale sólo para permitir la entrada del perro lazarillo de este caso en concreto, sino para permitir en general

${ }^{23}$ Hasta que el legislador, si es que así ocurre, modifique la formulación deóntica de la regla. 
la entrada de perros lazarillos en restaurantes, es decir, el balance de razones opera sobre la clase de casos en que una persona ciega quiere entrar a un restaurante acompañada por un perro lazarillo. Además, el guardia intermedio reformula la regla para que ésta sea conforme al balance de razones. Esto querría decir que la regla queda reformulada del siguiente modo: "prohibida la entrada de perros salvo que estén adiestrados para guiar y convivir con personas".

Antes de pasar a ver algunas posibles objeciones a mi modelo de toma de decisiones, que me servirán para detallar la propuesta y dejar ver cuáles son sus partes más originales, querría hacer una serie de consideraciones relacionadas con el guardia que aplica mi modelo.

(i) Parte del problema que revela la existencia de casos recalcitrantes deriva del hecho de que, al dar expresión a la regla mediante la formulación deóntica, difícilmente se podrá capturar todo el alcance fáctico del balance de razones, i. e. el rango exhaustivo de situaciones fácticas en las que se aplica ese balance de razones. De hecho, muchas veces el legislador, al hacer el balance de razones, no piensa - probablemente no puede pensar - en todos los casos a los que alcanza ese balance de razones. Y esta dificultad - 0 , directamente, imposibilidad-se refleja, ciertamente, en la formulación deóntica.

Así, el decisor, el guardia, parece estar en una mejor posición epistémica que el deliberador o el legislador para conocer el alcance fáctico exhaustivo del balance de razones. El legislador que inicialmente hizo la ponderación de razones, como decía, no puede conocer con exactitud, y de forma exhaustiva, el conjunto de circunstancias fácticas sobre el que se aplicará ese balance de razones, y, en consecuencia, el antecedente del condicional deóntico, establecido por el propio legislador, no podrá reflejar de forma exhaustiva el conjunto de casos sobre los que opera el balance de razones. Pero de esto no se sigue que el balance de razones, cuantifique sobre casos particulares. Ciertamente, de ello tampoco se sigue que cuantifique sobre casos generales; pero el punto aquí es empezar a refutar la idea según la cual de una tesis epistémica -i. e. no se puede conocer el alcance fáctico exhaustivo del balance de razones, sino en el caso 
particular - no se sigue, no al menos por sí sola, una tesis lógica -i.e. el balance de razones cuantifica sobre casos particulares- ni metafísica -i. e. la particularidad es una propiedad metafísica del balance de razones-. Se trata, creo, de dos discusiones independientes: por un lado, cuál es la mejor posición epistémica para identificar el alcance fáctico del balance de razones y, por otro lado, cuál es el estatus lógico o metafísico del balance de razones. Más tarde, en las objeciones, volveré sobre esto.

En todo caso, para el modelo intermedio el antecedente del condicional deóntico, en virtud de la mejor posición epistémica del decisor, resulta cada vez más refinado o más especificado, ${ }^{24}$ aunque esto no significa necesariamente que el antecedente de la regla quede completamente clausurado para siempre.

(ii) El guardia intermedio no entra a valorar cada vez si la decisión arrojada por la regla general es consistente con el balance de razones que justifica la regla. El guardia intermedio sólo entra a valorar el balance de razones si se encuentra con un caso particular en el que, además de los hechos explícitamente previstos por la regla, concurren otros hechos no previstos en el antecedente de la regla. Y cuando esto ocurre, si el alcance fáctico del balance de razones se proyecta también sobre ese caso particular, que es justamente lo que tiene que descubrir el decisor, este último modifica la formulación deóntica refinando su antecedente. Y si el balance de razones no alcanza a ese caso, entonces no modifica la regla y simplemente extrae la consecuencia normativa indicada por la regla. En la medida en que el antecedente se va refinando $-i$. $e$. quedan regulados más conjuntos de hechos posibles-, más probabilidades hay de que el guardia no tenga que valorar la ponderación de razones y se limite únicamente a aplicar la regla. Así pues, a diferencia del guardia moldeado según el modelo basado en reglas, que nunca valora la ponderación de razones, y a diferencia también del guardia particularista, que siempre valora la ponderación de razones, ${ }^{25}$ el guardia intermedio unas veces entra a valorar la

24 Moreso, J. J., La Constitución: modelo para armar, Madrid, Marcial Pons, 2009.

25 El guardia particularista "entra, cada vez, a valorar. Sopesa, cada una de las veces, las razones que se aplican; $y$, si hay una regla, decide caso por caso, sobre la base de este examen, si se debe aplicar o no" (Celano, 2015: 160 y 161). 
ponderación del caso particular — cuando concurren circunstancias no previstas en el antecedente de la regla-y otras veces no - cuando concurren sólo las circunstancias previstas en el antecedente de la regla-. ${ }^{26}$

(iii) El RoL, entonces, está efectivamente comprometido con la generalidad del razonamiento jurídico-práctico, pero no en el nivel de la formulación deóntica, sino, primariamente, en el nivel de las razones. Aunque lo anterior es un poco inexacto: no se trata de que rechace el carácter general de las reglas. Lo que ocurre es que pone el énfasis en el carácter general del balance de razones, sin excluir la posibilidad de formular las reglas mediante el cuantificador universal, pero a sabiendas de la limitación mencionada en (i). Así, el RoL debería estar comprometido con la concepción intermedia de la toma de decisiones, completando de este modo el modelo estándar de razonamiento jurídico-práctico afirmando, por un lado, que es contingente que el guardia deba evaluar cada vez si la solución normativa derivada de la regla es consistente con el balance de razones $y$, por otro lado, que el balance de razones es general.

\section{OBJECIONES}

\section{Disminución de la previsibilidad}

Esta objeción, inspirada en las virtudes que instancia el modelo de toma de decisiones basado en reglas, se puede plantear en los siguientes términos: si el decisor —el guardia o el juez- puede alterar la solución normativa porque le está permitido controlar la consistencia entre la solución normativa y el balance de razones, entonces la previsibilidad de sus decisiones disminuye. Con el modelo intermedio, tenemos menos certeza que con el modelo basado en reglas.

26 Alguien podría alegar que justamente cuando las circunstancias concurren o cuando no lo hacen es lo que está en juego. Esta es una impugnación potente. Intentaré responder a ella en la sección de objeciones. 


\section{RULE OF LAW Y CASOS RECALCITRANTES}

Me parece que la manera correcta de responder a esta objeción es, para empezar, aceptándola. Sin embargo, creo que hay una consideración de orden superior que justifica esa disminución de la previsibilidad. Para intentar dar cuenta de esta consideración, desarrollaré lo que voy a denominar "la paradoja de la inestabilidad política del modelo basado en reglas".

Imaginémonos un sistema jurídico en que a los jueces sólo les está permitido tomar decisiones sobre la base de las reglas emanadas por los órganos legislativos sin considerar nunca el balance de razones que justifica esas reglas; es decir, imaginémonos un sistema que adopte el modelo basado en reglas. En este escenario, dada la previsibilidad de las decisiones se presume que el sistema sería políticamente estable. ¿Por qué? Porque todos podrían tomar decisiones de forma autónoma, ya que todos sabrían qué está permitido y qué no. Una vez asegurada la autonomía individual, no habría razones para que los ciudadanos hicieran que el sistema fuera inestable. 0 así dice la teoría.

Sin embargo, como decía al presentar el dilema más arriba, en el escenario planteado en el párrafo anterior tal aumento de la previsibilidad iría en detrimento de la justicia, ya que al estarle prohibido al juez la valoración de la consistencia entre la solución normativa aportada por la regla y el balance de razones que justifica ésta, la probabilidad de que se dieran soluciones que consideramos incorrectas sería alta. ¿Cuán alta? Aquí conviene tener en mente algo que he dicho anteriormente: el legislador, al formular la regla, no tiene - probablemente no puede tener- en cuenta el alcance fáctico exhaustivo del balance de razones, por lo que la formulación deóntica de ese balance de razones difícilmente capturará ese alcance fáctico del balance de razones de forma exhaustiva. En esta tesitura, los casos recalcitrantes aumentarán. Y un sistema sobre el cual no sólo siempre planea la sombra de los casos recalcitrantes - algo que como veremos también le ocurrirá a un sistema que adopte el modelo intermedio, y que difícilmente se puede evitar si es que queremos alcanzar un grado razonable de justicia-, sino que muy frecuentemente arroja casos recalcitrantes, no será un sistema estable, porque aunque los ciudadanos tengan garantizada la autonomía individual, no parece que esto vaya a ser lo único que les pre- 
ocupe. Hay otras consideraciones morales, además de esa noción (más bien austera) de autonomía individual, que preocupan a los ciudadanos, y alguna forma de equidad se cuenta entre ellas. ${ }^{27}$ Así que resulta más bien optimista pensar que la satisfacción de la autonomía individual garantiza la estabilidad política del sistema jurídico. Si el sistema jurídico, aún asegurando la autonomía individual, no ofrece algún grado relativamente elevado de justicia sustantiva de forma sistemática, es razonable pensar que la estabilidad política de ese sistema se verá reducida. Así, parece que lo necesario, en términos generales, para la estabilidad de un sistema es una especie de equilibrio entre la satisfacción de la autonomía individual y la satisfacción de la expectativa de que el sistema aporte cierto grado de justicia sustantiva. Y el modelo intermedio parece mejor pertrechado que el modelo basado en reglas para acercarse a ese punto de equilibrio, ya que, como hemos visto, el modelo basado en reglas pone todo el énfasis en la garantía de la autonomía individual. La estabilidad del sistema, sin embargo, parece no depender únicamente de ese valor. ${ }^{28}$

2. ¿Cuándo puede decirse que concurren otros hechos, además de los explícitamente mencionados en el antecedente de la regla?

Según el modelo intermedio que yo he delineado, el guardia sólo entra a valorar el balance de razones si se encuentra con un caso

27 Haidt, J., The Righteous Mind. Why Good People are Divided by Religion and Politics, Penguin, 2011, pp. 150 ss., y Berlin, I., The Crooked Timber of Humanity, 3a. ed., Princeton University Press, 2013, cap I.

28 No hace falta, para esta discusión, adoptar ninguna postura metanormativa específica, pues aquí no se asume ningún estatus ontológico ni tampoco el carácter veritativo-funcional de los juicios normativos, ni siquiera del balance de razones; únicamente se asume que es posible controlar racionalmente la consistencia entre un dado balance de razones y la solución arrojada por una regla formulada a través de un cuantificador universal. Quizá únicamente desde un emotivismo primitivo, con una semántica muy poco desarrollada en relación con enunciados que contienen términos no-descriptivos, se puede poner en duda la posibilidad conceptual de ese juicio de consistencia entre el balance de razones y la solución normativa que aporta la regla. 
particular en el que, además de los hechos explícitamente previstos por la regla, concurren otros hechos no previstos en el antecedente de la regla.

La objeción de esta subsección viene a decir que, en realidad, aunque es lógicamente posible que los hechos explícitamente previstos por la regla aparezcan solos, la mayor parte de las veces esos hechos suelen ir acompañados de otros muchos hechos no mencionados en el antecedente. De facto, pues, el decisor siempre, o casi siempre, tendría que acudir al balance de razones para valorar cuál es la decisión en ese caso particular, con lo que no habría ninguna diferencia con el decisor particularista.

Lo que se necesita para bloquear esta objeción es poder mostrar que habrá algunos hechos nuevos que obligarán a acudir al balance de razones, y otros hechos nuevos que no dispararán la necesidad de acudir al balance de razones.

Pero la objeción proseguiría del siguiente modo: distinguir entre esos dos supuestos no es nada más que ver si el balance de razones cuantifica o no sobre ese conjunto de hechos no explícitamente mencionados por la regla. Es el alcance del balance de razones lo que convierte o no en relevantes esos hechos nuevos. Como consecuencia, "mi" decisor, al igual que el particularista, tendría que acudir al balance de razones en todos los casos. No habría, insistiría el autor de esta objeción, ninguna diferencia entre el guardia particularista y el guardia intermedio. ${ }^{29}$

Es cierto, sin lugar a dudas, que lo dicho en el párrafo anterior es posible; esto es, se puede determinar qué hechos son relevantes en función de cuál es el alcance fáctico del balance de razones. Sin embargo, el derecho es una práctica social, y, en consecuencia, qué hechos son (por lo menos) potencialmente relevantes no depende únicamente - ni siquiera, me atrevería a decir, primordialmente- del balance de razones. El derecho, como dice Bayón, es

${ }^{29}$ Como intentaré mostrar a continuación, creo que hay una diferencia sustancial. Pero si mi argumento fallara, seguiría existiendo una diferencia lógica o metafísica entre el modelo intermedio y el modelo particularista, a saber: el balance de razones es generalista para el modelo intermedio y particularista para el modelo particularista. 
"convencional, ...consiste en un conjunto de creencias compartidas y de actitudes y expectativas interdependientes constitutivas de una práctica social". ${ }^{30}$ Ese conjunto de creencias y actitudes, denominémoslo convención jurídica, determina, al menos en parte, qué hechos son en general potencialmente relevantes para el derecho. ${ }^{31} \mathrm{Se}$ trata de un punto complicado en la teoría del derecho, ${ }^{32}$ pero a los efectos de la respuesta a esta objeción creo que basta con reconocer la existencia de una convención jurídica, que tiene cierta fuerza normativa, ${ }^{33}$ que prescribe, de modo general, cuándo los hechos de un caso pueden ser potencialmente relevantes en la adjudicación de un caso particular.

Así, el decisor necesitará acudir al balance de razones para hacer el juicio de consistencia cuando haya hechos nuevos y éstos sean considerados potencialmente relevantes por parte de la convención jurídica. Por ejemplo, imaginémonos dos supuestos: 1) un señor ciego acompañado de un perro lazarillo quiere entrar en el restaurante; 2) un señor, en perfecto estado de salud, quiere entrar al restaurante acompañado de un perro que lleva un corte de pelo del símbolo de la paz. Asumamos que la regla que está en juego aquí es: "prohibida la entrada de perros en el restaurante". La convención jurídica considera como potencialmente relevante el hecho de que el perro del señor ciego sea un perro lazarillo, y el decisor de turno, sobre la base de esa potencial relevancia, acudirá a explorar el balance de razones que justifica la regla mencionada. De modo con-

30 Bayón, J. C., "Derrotabilidad, indeterminación del derecho y positivismo jurídico", Isonomía, 13, octubre de 2000, pp. 110 y 111.

31 Rodríguez y Sucar consideran que la identificación del derecho (i. e. el contenido de significado de las formulaciones normativas) se desarrolla apelando a las reglas semánticas vigentes en la comunidad de hablantes ordinarios, pero también apelando a las convenciones interpretativas fruto de la práctica resultante entre los operadores jurídicos (Rodríguez y Sucar, 1998, citados en Bayón, 2000: 103). De un modo parecido, qué hechos pueden ser potencialmente relevantes en un caso depende, al menos en parte, de ese tipo de convenciones, sólo que aplicadas no al contenido de significado de las formulaciones normativas.

32 Aunque como dice Bayón, parece ser aceptado por todo el mundo (Bayón, 2000: 103).

33 En el sentido hartiano de que los operadores jurídicos adoptan una actitud crítico-reflexiva hacia ella. 
trario, el hecho de que un perro esté rasurado de modo tal que lleve el símbolo de la paz no es, en general, considerado potencialmente relevante por parte de la convención jurídica, y por lo tanto no se dispararía la necesidad de acudir al balance de razones. ${ }^{34}$

Antes de pasar a la siguiente objeción, quisiera hacer algunas observaciones.

En primer lugar, el hecho de que la convención jurídica considere potencialmente relevantes los hechos no significa que entonces esos hechos pasen a figurar automáticamente en el antecedente de la regla; que sean potencialmente relevantes quiere decir que disparan la necesidad del decisor de explorar cuál es el alcance fáctico del balance de razones. Pero puede ocurrir que haya hechos potencialmente relevantes que disparen esa obligación, y que al final, cuando el decisor ha explorado el balance de razones, descubra que no son hechos relevantes para la solución del caso particular, $\mathrm{y}$, por lo tanto, concluya que no deben figurar en el antecedente de la regla.

En segundo lugar, nótese que a veces puede existir superposición entre aquellos hechos que la convención jurídica considera potencialmente relevantes y aquellos que de hecho lo son con arreglo al balance de razones. Pero ello será contingente: algunas veces habrá superposición, otras no. ${ }^{35} \mathrm{He}$ aquí pues la diferencia entre el decisor intermedio y el particularista: el primero sólo estará obligado a acudir al balance de razones cuando haya hechos nuevos y éstos sean considerados potencialmente relevantes por parte de la convención jurídica, mientras que el segundo siempre está obligado a acudir al balance de razones, incluso aun cuando la convención jurídica considere que los eventuales hechos nuevos son irrelevantes jurídicamente hablando. Esto quiere decir que es probable que el conjunto de decisiones tomadas por el guardia intermedio con-

34 Desde luego, un operador jurídico, o unos pocos, pueden impugnar lo que acabo de decir en el texto, pero me parece que estaríamos todos, o casi todos, de acuerdo en que ese operador o esos pocos operadores se estarían situando, al menos respecto de este punto, fuera de la convención jurídica.

35 También será contingente qué hechos serán potencialmente relevantes, en el sentido de que ello puede cambiar en función de cuáles sean las personas —así como sus "ideologías" - que constituyen esa convención jurídica. 
tenga menos justicia que el del decisor particularista, pues el grado mayor de justicia posible lo tenemos cuando acudimos en todos y cada uno de los casos al balance de razones. ${ }^{36}$

En tercer lugar, y a modo de extensión de lo dicho en el párrafo anterior, uno - el autor de esta objeción, por ejemplo- podría preguntarse: pero ¿por qué no ignorar lo que diga la convención jurídica y obligar al decisor a acudir siempre al balance de razones? ¿Por qué no ir por el grado más alto posible de justicia? Y la respuesta es que eso implicaría un grado bajo (potencialmente incluso muy bajo) de certeza, y el derecho, para contribuir a asegurar la autonomía individual, debe ser, en buena medida, previsible. Por lo demás, esta preferencia por un equilibrio entre certeza y justicia funciona, si es que funciona, únicamente en relación con el razonamiento jurídico-práctico; pero no estoy seguro de que funcione en cualquier ámbito práctico, singularmente en el de la moral. ¿Por qué? Por un lado, porque aun suponiendo que exista en el reino de la moral una convención acerca de qué hechos son moralmente relevantes para reconsiderar nuestro balance de razones morales, no me parece obvio que se pueda identificar esa convención sin la existencia de una autoridad normativa supraindividual, algo que no se da en el reino de la moral, pero sí existe en el derecho (los operadores jurídicos no tienen un equivalente en el dominio moral, no al menos en el dominio moral laico), y, por otro lado, en el reino de la moral, me parece que tiene prioridad la búsqueda de la justicia, mientras que en el derecho, o por lo menos en algunos ámbitos del derecho, buscamos un punto de equilibrio entre justicia y certeza o previsibilidad. ${ }^{37}$

36 Digo que es probable porque me parece difícil que haya siempre superposición entre lo que la convención jurídica considera como potencialmente relevante y lo que el balance de razones convierte en relevante. En este sentido, quizá sea razonable decir que los operadores jurídicos -i. e. la convención jurídica- pueden cometer errores.

37 Así pues, mi tesis es en buena parte normativa: dado que preferimos que en el derecho haya un equilibrio entre certeza y justicia, el modelo que mejor parece alcanzar ese equilibrio es el del modelo intermedio. Alguien podría entonces preguntarme: ¿y por qué se debería activar la obligación de acudir al balance de razones cuando la convención jurídica así lo indique? Y mi respuesta es que, como señalaba 
En cuarto lugar, no hay clausura del antecedente de la regla. No estoy ni asumiendo ni intentado mostrar que existe lo que Alchourrón y Bulygin llaman "tesis de relevancia última", o lo que Jorge Rodríguez denomina "regla de clausura última". Puede que en un sistema jurídico exista tal regla o no, como bien dicen Rodríguez y Sucar. De todos modos, y aquí estoy saliendo de mi zona de confort, quizá podría decirse que en mi modelo existe algo así como una tesis de clausura última respecto del balance de razones; ocurre, sin embargo, que no es posible descubrir cuál es la regla de clausura, por razones epistémicas, sino ex post facto. Sea como sea, en mi modelo, como he dicho ya anteriormente, no hay clausura del antecedente de la regla, elemento que, a mi juicio, siempre queda abierto, pues como decía Hart, siempre somos relativamente ignorantes respecto de los hechos futuros. ${ }^{38}$

\section{El balance de razones es particularista}

Según el particularismo, "puede suceder que en otro caso del mismo tipo lo que aquí (i. e. en el caso particular actual) es una razón para hacer $A$, no sea una razón para hacer $A$ o sea una razón para no hacer $A " .^{39}$ Así, para el particularista es posible identificar, en los casos particulares, ciertos hechos como razones para actuar de un determinado modo. Pero el particularista niega que esas razones se proyecten (necesariamente) hacia el futuro, hacia otros casos; las razones son particularistas, no generalistas.

Nótese que en esta presentación del particularismo se habla de razones, no de balance de razones. Sin embargo, no veo por qué deberíamos rechazar que esa misma tesis se aplique al balance de razones. Así pues, el balance de razones $B R$, por ejemplo, cuantifica

Bayón, el derecho está fundado sobre unas expectativas interdependientes constitutivas de una práctica social (Bayón, 2000: 111), por lo que tiene sentido que el criterio acerca de cuándo acudir al balance de razones esté basado, por lo menos en alguna medida, en ese conjunto de expectativas interdependientes.

38 Hart, H. L. A., The Concept of Law, 3a. ed., OUP, 1961, p. 128.

39 Celano, B., Derecho, justicia, razones. Ensayos 2000-2007, Madrid, Centro de Estudios Políticos y Constitucionales, 2009, p. 441. 
sobre el caso particular $t$ para llevar a cabo la acción $A$; pero podría ocurrir que, en el caso particular $t^{\prime}$, aun siendo del mismo tipo que $t$, el balance $B R$ indique la acción no- $A$.

Así pues, lo que sostiene el particularista es que el balance de razones se proyecta únicamente sobre casos particulares, pero no sobre casos generales. Sin embargo, no parece tener mucho sentido aceptar la idea de que el balance de razones es particularista si ello implica asumir que el dominio de las razones, y, más específicamente, el reino del razonamiento práctico, es ininteligible. ¿Por qué digo esto? La inteligibilidad de las razones, y del balance de razones, quiere decir que debe existir alguna diferencia entre dos supuestos de hecho que explique por qué el balance de razones $B R$ indica $A$ en un supuesto, pero no- $A$ en otro (Raz, 2000: 230). Esto parece implicar que el dominio de las razones es inteligible cuando o bien (1) un balance de razones indica la misma acción para dos (o más) casos particulares que son instancias del mismo tipo de supuesto de hecho, o bien (2) un balance de razones indica acciones distintas (e incluso contradictorias entre sí) para casos particulares cuando éstos no son instancias del mismo tipo de supuesto de hecho. Llamaremos a este requisito, siguiendo a Raz, "el requisito de la inteligibilidad de las razones".

Creo que resulta evidente que la presentación del particularismo hecha al principio de esta subsección no puede satisfacer el requisito de inteligibilidad de las razones. ${ }^{40}$ De hecho, parece que la inteligibilidad de las razones empuja hacia alguna forma de generalismo. ${ }^{41}$

Así, aceptar la tesis particularista significaría aceptar que la empresa del razonamiento práctico es ininteligible. Y este parece un precio demasiado alto si lo que se quiere es, precisamente, de-

40 Estrictamente hablando, esta forma de particularismo parecería estar comprometida con la imposibilidad misma del razonamiento práctico, pues simplemente no habría nada que uniera una conclusión normativa con otras, todo sería meramente arbitrario, pues dependería del acaecimiento aleatorio de los hechos de cada caso particular (Celano, 2009: 444).

41 Raz, J., Engaging Reason. On the Theory of Value and Action, OUP, 2000, pp. 220-235. 
linear el razonamiento práctico, algo que el particularista no parece, al menos a primera vista, estar dispuesto a rechazar (ya que el particularista - o por lo menos Celano- no parece dispuesto a rechazar la idea de que cuando tomamos decisiones prácticas razonamos). Si queremos satisfacer el requisito de la inteligibilidad, deberíamos abrazar alguna forma de generalismo, ya sea la del guardia cuyas decisiones están basadas en reglas o la del guardia intermedio.

¿Pero es esto así? ¿Abandonará la batalla así de fácil el particularista?

Creo que no. Uno puede imaginar otra forma de particularismo que base su objeción acerca de cuándo dos —o más- casos son del mismo tipo. El argumento funcionaría más o menos así: para que la propuesta generalista (tanto la del guardia intermedio como la del guardia de las reglas) fuera operativa sería necesario poder afirmar, por avanzado, que dos casos son del mismo tipo. ${ }^{42} \mathrm{Y}$ esto es lo que el particularista niega que pueda obtenerse.

Dos casos son del mismo tipo si y sólo si comparten todas las propiedades relevantes. Y ¿qué afirma el particularista a este respecto?

No es posible delimitar previamente el conjunto de propiedades relevantes... Sería magnífico si tuviéramos a disposición una tesis de relevancia no sujeta a ampliaciones o revisiones (tesis de relevancia última): un listado, cerrado y exhaustivo, de las propiedades relevantes para la decisión sobre cómo comportarse -o sobre qué cosa es una razón para hacer algo- en todo caso posible. ${ }^{43}$

Desafortunadamente, prosigue el particularista, ese listado no existe. Sin embargo, el particularista no descarta que no se puedan identificar las propiedades relevantes de un caso particular. Lo que descarta es que se puedan identificar con anterioridad al acaecimiento del caso particular, es decir, descarta una manera de concebir la idea de caso general, i. e. la idea del listado cerrado y exhaustivo de las propiedades relevantes. Esto, según el particula-

42 Celano, 2009, pp. 44 y ss.

${ }^{43}$ Celano, B., Derecho, justicia, razones. Ensayos 2000-2007, Madrid, Centro de Estudios Políticos y Constitucionales, 2009, p. 443. 
rista, no significa que no se puedan hacer generalizaciones a partir de un caso:

Proyecciones o extrapolaciones de casos pasados (o actuales) hacia casos futuros (o posibles) son admisibles. Pero nada nos garantiza que den en el blanco - nada garantiza que no lleguen a reclamar inesperadamente, una revisión, a la vista de circunstancias que están fuera de lo común. ${ }^{44}$

Reformulada de este modo, la objeción particularista, además de poder dar cuenta del requisito de inteligibilidad, parece poner el énfasis en la imposibilidad de conocer ex ante cuáles son las propiedades relevantes de un caso general; las propiedades relevantes de un caso general sólo pueden ser objeto de conocimiento ex post y, de cualquier manera, el conjunto de propiedades relevantes que componen el caso general siempre estará sujeto a ser modificado si el caso particular así lo exige.

¿Es esta una objeción para el generalismo? Creo que, efectivamente, es una objeción insalvable para la concepción del razonamiento práctico sobre la que se apoya el guardia cuyas decisiones se basan en el modelo de reglas. Pero creo que no lo es para el denominado guardia intermedio. Veamos por qué.

La objeción particularista afirma que no es posible conocer de antemano cuáles son las propiedades relevantes de un caso. Pero en mi delineación del guardia intermedio, esta posibilidad se da por descontada: el decisor siempre está en mejores condiciones epistémicas para saber cuál es el alcance fáctico del balance de razones. Nótese que la objeción particularista reformulada parece poner el énfasis en dos puntos: por un lado, afirma una suerte de tesis epistémica, según cual el conocimiento de las propiedades relevantes, y de cómo éstas condicionan la respuesta normativa, tiene naturaleza ex post, y, por otro lado, también afirma la imposibilidad de una tesis de relevancia última para las reglas. Pero ninguno de estos dos puntos es incompatible con la idea de que el balance de razones sea general. El primer punto es epistémico, y no afectaría al estatus (ge-

44 Ibidem, p. 444. 
neral o particular) de las razones, $\mathrm{y}$, traducido al nivel de las razones, "sólo" nos dice que en sentido estricto no se puede saber el alcance fáctico del balance de razones sino en los casos particulares. Pero la concepción que yo presenté más arriba ya había admitido esto. Con el segundo punto sucede algo similar: se refiere a la imposibilidad de encapsular de forma exhaustiva en un condicional deóntico (i. e. una regla) el conjunto de propiedades relevantes de un caso general. Pero mi modelo también había admitido este extremo.

Nótese, además, que este segundo punto se refiere a la tesis de relevancia última de una regla, no de un balance de razones. Y es que el balance de razones, con arreglo a la delineación que yo he hecho de él, no contiene de forma explícita y exhaustiva su alcance fáctico, algo que no tendría sentido hacer, dado que ya hemos admitido que el decisor está en mejor posición epistémica para saber cuál es el alcance epistémico de aquél.

No querría sugerir que estos contraargumentos son concluyentes. Quizá el balance de razones es particularista, pero los argumentos particularistas hasta el momento disponibles no eliminan la posibilidad de que el balance sea generalista.

\section{CoNCLUSIÓN}

Las conclusiones de este artículo pueden ser sintetizadas en las siguientes consideraciones:

Hay espacio lógico entre un modelo de razonamiento jurídicopráctico rígidamente basado en reglas y un modelo particularista. Este es el espacio que "mi" modelo intermedio busca explotar.

No sólo hay espacio lógico para el modelo intermedio, sino que, dada la búsqueda de un equilibrio entre justicia y certeza que parecemos exigirle al derecho, hay buenas razones normativas para abrazar el modelo intermedio que yo he delineado aquí.

El RoL no necesita desprenderse de su compromiso con la generalidad del dominio jurídico-práctico para hacer frente al desafío de los casos recalcitrantes. 
VI. BIBLIOGRAFÍA

Alchourrrón, Carlos, "Detachment and Defeasibility in Deontic Logic", Studia Logica: An International Journal for Symbolic Logic, 1996, vol. 57, núm. 1.

Alchourrón, Carlos y Bulygin, E., Normative Systems, Verlag, 1971.

BARBERIS, M., "Possono governare, le leggi? Il dilemma del rule of law”, en Pino, G. y Villa, V. (eds.), Rule of Law. L'ideale della legalità, Bologna, Il Mulino, 2016.

BAYón, J. C., "Derrotabilidad, indeterminación del derecho y positivismo jurídico", Isonomía, 13 de octubre de 2000.

Berlin, I., The Crooked Timber of Humanity, 3a. ed., Princeton University Press, 2013.

CAmpbell, T., The Legal Theory of Ethical Positivism, Aldershot/ Brookfield/Singapore/Syndey, Darmouth, 1996.

Caracciolo, R., El derecho desde la filosofía, Madrid, CEPC, 2009.

Celano, B., Derecho, justicia, razones. Ensayos 2000-2007, Madrid, Centro de Estudios Políticos y Constitucionales, 2009.

Celano, B., "True Exceptions: Defeasibility and Particularism", en FERrer, J. y G. B. RATTI (eds.), The Logic of Legal Requirements: Essays on Defeasibility, OUP, 2012.

Celano, B., "Publicity and the Rule of Law", in Leiter, B. y Green, L., (eds.), Oxford Studies in Philosophy of Law, OUP, 2013.

Celano, B., "Rule of Law y particularismo ético", en Luque, P. (ed.), Particularismo. Ensayos de filosofía del derecho y filosofía moral, Madrid, Marcial Pons, 2015.

DANCY, J., “¿Qué es el particularismo en ética”, en Luque, P. (ed.), Particularismo. Ensayos de filosofía del derecho y filosofía moral, Madrid, Marcial Pons, 2015.

DWorkin, R., Taking Rights Seriously, HUP, 1977.

Dworkin, R., Law's Empire, HUP, 1986.

Fuller, L., The Morality of Law, New Haven, Yale University Press, 1969. 
HAIDT, J., The Righteous Mind. Why Good People are Divided by Religion and Politics, Penguin, 2011.

Hart, H. L. A., The Concept of Law, 3a. ed., OUP, 1961.

Kramer, M., Objectivity and the Rule of Law, CUP, 2007.

LAPORTA, F., El imperio de la ley. Una visión actual, Madrid, Trotta, 2007.

Maccormick, N., Rhetoric and the Rule of Law, OUP, 2005.

Moreso, J. J., La Constitución: modelo para armar, Marcial Pons, 2009.

Pino, G., "Legalità penale e Rule of Law”, en Pino, G. y Villa, V. (eds.), Rule of Law. L'ideale della legalità, Bologna, Il Mulino, 2016.

Postema, G., "Il dominio del diritto", en Pino, G. y Villa, V. (eds.), Rule of Law. L'ideale della legalità, Bologna, Il Mulino, 2016.

RAZ, J., The Authority of Law, OUP, 1979.

RAZ, J., Engaging Reason. On the Theory of Value and Action, OUP, 2000.

RAz, J., "The Problem of Authority: Revisiting the Service Conception", The Minnesota Law Review, 2006.

Redondo, C., "Legal Reasons: Between Universalism and Particularism", Journal of Moral Philosophy, vol. 2, 2005.

Rodríguez, J. L. y SucAR, G., "Las trampas de la derrotabilidad. Niveles de análisis de la indeterminación del derecho", Analisi e diritto, 1998.

RoDRÍGUEZ, J. L., "Normas y razones: un dilema la irracionalidad y la irrelevancia”, Revista Jurídica de la Universidad de Palermo, año 13, núm. 1, diciembre de 2012.

Schauer, F., "Formalism", The Yale Law Journal, vol. 97, núm. 4, 1988.

Schauer, F., Playing by the Rules. A Philosophical Examination of Rule-Based Decision-Making in Law and in Life, OUP, 1991.

Schauer-LandaU, R., "Moral Rules", Ethics 107 (July), 1997.

Sunstein, C., One Case at a Time. Judicial Minimalism on the Supreme Court, HUP, 2001. 
Tamanaha, B., "A Concise Guide to the Rule of Law", en PalombeLLA, G. y WaLKer, N. (eds.), Relocating the Rule of Law, Hart, Oxford, 2009.

Von Hayer, F., The Constitution of Liberty, Chicago, Chicago University Press, 1969.

WALDron, J., "The Concept and the Rule of Law”, Georgia Law Review, 43, 2008.

WALDron, J., "Stare Decisis and the Rule of Law: A Layered Approach", 111 Michigan Law Review 1, 2012.

Waldron, J., "How Law Protects Dignity", Cambridge Law Journal, 71, 2a. ed., 2012. 